\title{
Treatment plan dosimetric impact after catheter stretch during multi-catheter interstitial breast brachytherapy
}

\author{
Eeva Boman, PhD, Dean Paterson, BRT (Hons), Junou Huang, BRT, Shelley Pearson, BRT, \\ Carol Johnson, FFRad.T(SA), FRANZCR \\ Radiation Treatment Department, Blood and Cancer Centre, Wellington Hospital, Wellington, New Zealand
}

\begin{abstract}
Purpose: Nylon 6/6 interstitial brachytherapy catheters may stretch when exposed to moisture, mechanical tension, and body temperature. The purpose of this study is to evaluate the magnitude of catheter stretch during a course of multi-catheter interstitial breast brachytherapy for nylon 6/6 treatment catheters, and to assess the impact this has on treatment plan dosimetry.

Material and methods: Catheters were exposed to water at $37^{\circ} \mathrm{C}$ for six days and the internal catheter length change $\left(\Delta L \_W\right)$ was measured daily. Additionally, the measured internal catheter length change $\left(\Delta L \_P\right)$, performed prior to each treatment fraction, for seven interstitial breast brachytherapy patients (total of 76 catheters) were retrospectively analyzed. The maximum length change seen in $\Delta L_{-} P$ and $\Delta L_{-} W$ were introduced as a source positional error to retrospective treatment plans, and treatment plan dosimetry was analyzed.

Results: $\Delta L \_W$ and $\Delta L \_P$ were on average $+4.0 \%$ and $+1.6 \%$ of the catheter lengths in water or tissue after 48 hours. Weak correlation was seen between the average $\Delta L \_P$ per catheter and both the catheter length within tissue $(\rho=0.36$, $p=0.0007)$, and the mid-catheter depth in tissue $(\rho=0.42, p<0.0001)$. The $\mathrm{D}_{90 \mathrm{CTV}}$ decreased $1.5 \%(p<0.05)$ and $8.2 \%$ $(p<0.05)$ when the $\Delta L \_P$ and $\Delta L \_W$ were introduced to the initial plans.

Conclusions: Nylon 6/6 catheters stretch during a course of multi-catheter interstitial breast brachytherapy treatment. The observed stretch may affect treatment plan dosimetry, if the catheter internal length is only measured immediately after the insertion. Additional catheter length checks are recommended to verify the actual catheter internal length during the treatment.

J Contemp Brachytherapy 2017; 9, 5: 418-423 DOI: https://doi.org/10.5114/jcb.2017.70987
\end{abstract}

Key words: APBI, breast cancer, catheter stretch, interstitial, HDR, source positional error.

\section{Purpose}

Accelerated partial breast irradiation (APBI) using multi-catheter interstitial breast brachytherapy is an alternative to whole breast irradiation (WBI) following breast conserving surgery for selected patients with early stage breast cancer [1]. The advantages of APBI include reducing overall treatment time to one week or less, and reducing radiation exposure to healthy tissue such as lung, heart, skin, and breast [2]. Although various techniques can be used to deliver APBI, multi-catheter interstitial breast brachytherapy has the most mature follow-up data and allows excellent conformity and target coverage [3].

In 2015, the Groupe Européen de Curiethérapie and the European Society for Radiotherapy \& Oncology (GEC-ESTRO) published the 5-year results of a phase III, randomized, non-inferiority trial comparing WBI to multi- catheter interstitial APBI [4]. Multi-catheter APBI was found to be equivalent to WBI in terms of local control, disease-free survival, and overall survival, confirming the non-inferiority of the approach. In addition, the risk of grade 2-3 late side-effects to the skin was greater with WBI, and the risk of grade 2-3 subcutaneous tissue effects and grade 3 fibrosis were similar between the two techniques.

In 2016, our institution implemented a pilot project treating a small cohort of patients with high-dose-rate (HDR) multi-catheter interstitial breast brachytherapy in order to collect data, so that the long-term feasibility of the technique at our department could be assessed. Due to the relatively small target volumes, the positional accuracy of a HDR ${ }^{192}$ Ir brachytherapy source inside the treatment catheters is crucial in order to avoid under dosing the target volume and/or over dosing organs at 
risk. Prior to each brachytherapy treatment, the International Commission on Radiological Protection (ICRP) recommends manual insertion of a measurement wire to ensure that the total internal length of the transfer guide tube plus applicator/catheter equals the planned treatment length (internal applicator length measurement) [5]. This check also serves to identify any obstruction in the catheter or guide tube. The manufacturer of our ${ }^{192}$ Ir remote afterloader (VariSource iX, Varian Medical Systems, Palo Alto, CA, USA) endorses this recommendation, and it has been adopted as a local rule.

To the authors' knowledge, there have not been any studies describing how nylon 6/6 treatment catheters behave inside tissue, where they are exposed to moisture, mechanical tension, and body temperature. Our institutions initial experience of internal applicator length measurements for breast brachytherapy has shown that the nylon 6/6 treatment catheters used in our center appear to stretch over a patient 5-8 day treatment course. To the authors' knowledge, neither this phenomenon nor its consequences to the delivered dose has been reported in the literature. The purpose of this study is to evaluate the magnitude of catheter stretch during a course of multi-catheter interstitial breast brachytherapy, and to assess the impact of this on treatment plan dosimetry.

\section{Material and methods}

\section{Applicator internal length measurements in water}

Three unused interstitial catheters $(6.0 \mathrm{Fr} 30 \mathrm{~cm}$ single leader with filaments, Best Medical International, VA, USA), cut to $20 \mathrm{~cm}$ long, were exposed to water (submerged up to $15 \mathrm{~cm}$, with $5 \mathrm{~cm}$ protruding out of the water to simulate a clinical implant) and placed in an incubator (Panacea $2431 / \mathrm{V}$, Torre Piceadi, Italy) set to $37^{\circ} \mathrm{C}$ to investigate the internal catheter length change $\left(\Delta L_{-} W\right)$ when exposed to moisture and heat. The water in the incubator was left for 24 hours for the temperature to stabilize prior to the experiment. Additionally, three catheters were placed in air at $37^{\circ} \mathrm{C}$ (Panacea $2431 / \mathrm{V}$ ), and three in air at room temperature $\left(22^{\circ} \mathrm{C}\right)$ as a reference. Internal length measurements were carried out using a $150 \mathrm{~cm}$ measurement ruler and marker wire (Varian Medical Systems) without connecting the catheters to transfer guide tubes. The initial internal catheter lengths were measured prior to water and/or heat exposure, and repeated after exposure at 2 hours, 4 hours, 24 hours, and every 24 hours thereafter for 120 hours in total.

\section{Catheter insertion and treatment planning}

Plan and treatment data from the first seven APBI patients treated at our institution using multi-catheter interstitial brachytherapy were retrospectively analyzed. Hollow stainless steel trocars (Best Medical International) were implanted using a free-hand technique with the patient under general anesthesia. Intraoperative ultrasound (BK Flex Focus 500, BK Medical, Denmark) and C-arm (Siemens Arcadis Orbic, Siemens AG, Germany) imaging was used to guide the insertion. Catheter distribution was determined by a pre-implant computed tomography
(CT) (Philips Brilliance Big Bore, Philips Medical Systems, Fitchburg, WI, USA), and was designed in 2-4 planes with $1-1.5 \mathrm{~cm}$ applicator spacing to cover the target volume. The number of catheters per patient ranged from 8 to 18 , with 76 catheters implanted in total for the seven patients.

The trocars (Best Medical International) were exchanged for nylon $6 / 6$ treatment catheters $(6.0 \mathrm{Fr} 30 \mathrm{~cm}$ single leader with filaments, Best Medical International). Nylon buttons and friction cuffs were used to secure the catheters and no skin sutures were used. Catheters were cut so that approximately $5 \mathrm{~cm}$ of the proximal catheter was protruding from the patients' skin. The catheter internal lengths were not measured or known at this point.

The planning CT (Philips Brilliance Big Bore) was performed after the catheter insertion using $0.15 \mathrm{~cm}$ slice thickness. The clinical target volume (CTV) was contoured following the GEC-ESTRO Breast Cancer Working Group guidelines [3,6], and was cropped $0.5 \mathrm{~cm}$ from the skin surface and at the chest wall. The average volumes of the CTV and lumpectomy cavity were $59 \mathrm{~cm}^{3}$ (range, $32-111 \mathrm{~cm}^{3}$ ) and $7 \mathrm{~cm}^{3}$ (range, $9-21 \mathrm{~cm}^{3}$ ), respectively.

Initially, patients were prescribed 34 Gy in 10 fractions, bi-daily over 8-9 days (no treatment on the weekend). The three most recent patients were prescribed 32 Gy in 8 fractions, bi-daily over 5 days. At least 6 hours was required between each fraction. Only one fraction was delivered on the day of insertion.

Retrospective plans $\left(P_{R}\right)$ were developed using BrachyVision version 13.7 (Varian Medical Systems) with the HDR ${ }^{192} \mathrm{Ir}$ source $\left(\mathrm{V}_{\mathrm{S} 2000}\right.$, VariSource iX, Varian Medical Systems). Each retrospective plan was created by a single planner to ensure consistent plan quality. A $0.5 \mathrm{~cm}$ expansion on the CTV (dwell volume) was used to activate and set dwell positions using the "fit source positions inside structure" tool in BrachyVision (Figure 1). Volume optimization followed by individual dwell time optimization using the dose shaper tool was used to create clinically acceptable plans. The planning aims are presented in Table 1. Dose was calculated using the TG-43 dose calculation model and a $0.1 \mathrm{~cm}$ calculation grid. Skin

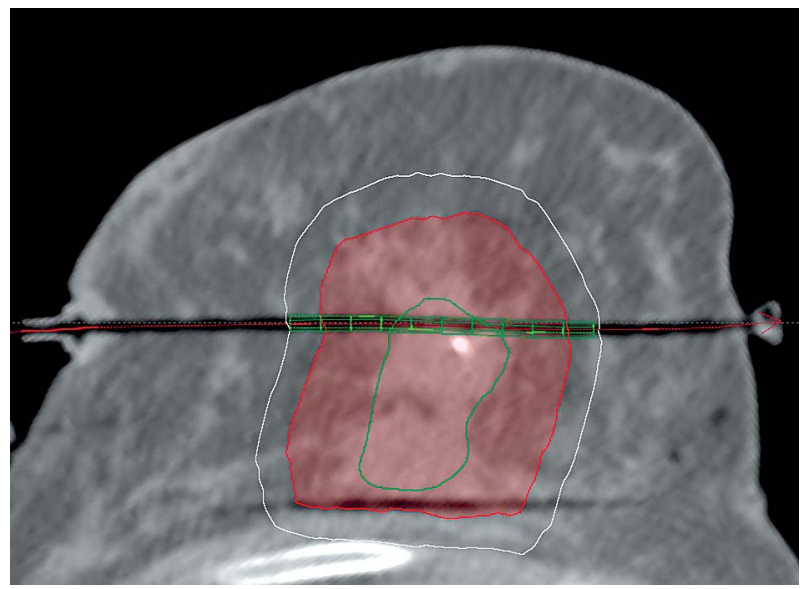

Fig. 1. Clinical target volume contour (red) with lumpectomy cavity (green). The dwell positions for one catheter are shown with the dwell volume (white), which is used to set the dwell positions 
Table 1. Average dose parameters $\pm \mathrm{SD}$ (min-max) with plan objectives for all patients for $P_{R}, P_{\Delta L_{-} P}$ and $P_{\Delta L}{ }_{-}$plans

\begin{tabular}{|c|c|c|c|c|}
\hline & Objectives & $P_{R}$ plans & $P_{\Delta L_{-} P}$ plans & $P_{\Delta L_{-} w}$ plans \\
\hline $\mathrm{D}_{90 \mathrm{ctV}}(\%)$ & $\geq 100$ & $101.1 \pm 6.0(90.2-109.4)$ & $99.6^{\star} \pm 5.3(89.9-107.1)$ & $92.9^{\star} \pm 3.9(83.9-97.0)$ \\
\hline$V_{100 \text { Body }}\left(\mathrm{cm}^{3}\right)$ & $<150$ & $82.0 \pm 32.0(43.2-137.3)$ & $81.9 \pm 32.0(43.2-137.0)$ & $81.6 \pm 30.7(47.1-141.0)$ \\
\hline$V_{150 \text { Body }}\left(\mathrm{cm}^{3}\right)$ & $<50$ & $29.1 \pm 10.4(15.7-45.7)$ & $29.4 \pm 10.8(15.7-46.7)$ & $29.0 \pm 10.2(14.8-46.6)$ \\
\hline$V_{\text {200Body }}\left(\mathrm{cm}^{3}\right)$ & $<20$ & $11.7 \pm 4.1(6.2-18.5)$ & $11.7 \pm 4.2(6.2-18.9)$ & $11.5 \pm 3.9(6.0-18.3)$ \\
\hline DNR & $<0.35$ & $0.36 \pm 0.07(0.30-0.49)$ & $0.36 \pm 0.07(0.30-0.48)$ & $0.36 \pm 0.06(0.29-0.48)$ \\
\hline $\mathrm{D}_{0.2 \mathrm{ccskin}}(\%)$ & $<100$ & $84.2 \pm 16.6(51.9-102.8)$ & $83.2 \pm 16.0(54.5-104.0)$ & $82.4 \pm 16.6(55.3-111.5)$ \\
\hline $\mathrm{D}_{0.1 \mathrm{ccRibs}}(\%)$ & $<90$ & $69.1 \pm 20.0(34.7-89.0)$ & $68.0 \pm 19.9(33.4-88.0)$ & $67.3 \pm 18.9(33.4-87.8)$ \\
\hline
\end{tabular}

${ }^{*} p<0.05$ between $P_{R}$ and $P_{\Delta L P}$ or $P_{R}$ and $P_{\Delta L W}$ plans at Wilcoxon signed ranks test and paired samples $t$-test, $D_{90 c t V}$ - percent of the prescription dose covering $90 \%$ of the CTV (clinical target volume), $V_{100 \%}, V_{150 \%}, V_{200 \%}$ - volume of the anatomic volume (body) receiving 100\%, 150\%, 200\% of the prescribed dose, DNR-dose non-uniformity ratio $\left(V_{100} / V_{50}\right), D_{0.2 c c}, D_{0.1 c c}-$ minimum dose to the most exposed $0.2 \mathrm{~cm}^{3}, 0.1 \mathrm{~cm}^{3}$ (skin, ribs)

was defined as a $0.5 \mathrm{~cm}$ internal margin structure from the skin surface.

\section{Applicator internal length measurements in patient}

The catheter internal length measurements were performed prior to each treatment using a $150 \mathrm{~cm}$ measurement ruler and marker wire (Varian Medical Systems) with the transfer guide tubes connected to the catheters. The initial pre-planning length measurements were verified by two qualified radiation therapists approximately 2 hours after catheter insertion. These values were used in the treatment plans. The tolerance for two independent internal length measurements was $0.1 \mathrm{~cm}$. If the two measurements differed by $0.1 \mathrm{~cm}$, the longer measurement was applied to the treatment plan. For subsequent fractions, only one pre-treatment internal length measurement per catheter was performed.

The pre-treatment catheter internal length measurements were analyzed and reported for each fraction. The length of the catheter inside the breast tissue $\left(L_{C-B}\right)$ and the catheter depth from the skin surface at midcatheter $\left(d_{\mathrm{S}-\mathrm{C}}\right)$ were also measured from the planning $\mathrm{CT}$.

\section{Catheter stretch applied to treatment plans}

The maximum length change $\left(\Delta L \_P\right)$ seen in length measurements for each catheter over each patient's treatment course was introduced to the $P_{R}$ plans $\left(P_{\Delta L_{P} P}\right)$ by shifting the source positions by $\Delta L \_P$ along the catheters towards the proximal end of the catheter (where the transfer guide tubes were connected). Similarly, the maximum $\Delta L \_W(\%)$ seen in catheters exposed to water (averaged over three catheters) was applied to the $P_{R}$ plans $\left(P_{\Delta L_{-} W}\right)$, in which the source positions per catheter were shifted by $\Delta L \_W(\%)^{*} L_{\mathrm{C}-\mathrm{B}}$. The dosimetric changes in CTV coverage, dose-non-uniformity ratio $\left(\mathrm{DNR}=\mathrm{V}_{150} / \mathrm{V}_{100}\right)$, and organs at risk (OAR) dose were analyzed.

\section{Statistical analyses}

Statistical analyses were performed using SPSS $\left(\mathrm{V}_{22}\right.$, IBM Corp., US) to test differences between dose parameters from the $P_{R}, P_{\Delta L_{-} P}$ and $P_{\Delta L_{-} W}$ plans. Kolmogorov-Smirnov test was used to test the data normality. Non-normality was considered if $p<0.05$. Both paired samples $t$-test and the Wilcoxon signed ranks test were performed for all data due to the small sample size. Statistical significance was considered when $p<0.05$ on both tests. The correlation between the average $\Delta L \_P$ per catheter and both $L_{\mathrm{C}-\mathrm{B}}$ and $d_{\mathrm{S}-\mathrm{C}}$ was tested using non-parametric two-tailed Spearman's $\rho$-test. Significant correlation $(\rho)$ was considered when $p<0.05$.

\section{Results}

\section{Applicator length measurements in water}

The average catheter internal length measurements for the three catheters in water and the six in air are presented in Figure 2A. An average $\Delta L_{-} W$ of $+0.6 \mathrm{~cm}$ (range, $0.5-0.7 \mathrm{~cm})$, which means $+4.0 \%(3.3-4.7 \%)$ stretch on the water submerged length $(15 \mathrm{~cm})$ was seen in catheters exposed to water at $37^{\circ} \mathrm{C}$ after 24 hours, and remained on average the same over the rest of the time. The $\Delta L_{-} W$ was on average $+0.2 \mathrm{~cm}(1.3 \%)$ after 2 hours of the catheters being exposed to water (at the time when the first internal length measurements for the patients were carried out). The length change for reference catheters in air at $37^{\circ} \mathrm{C}$ and $22^{\circ} \mathrm{C}$ was within $\pm 0.1 \mathrm{~cm}$.

\section{Applicator length measurements in patients}

The results for catheter internal length measurements for all patients are presented in Figure 2B-D. The initial measurements were carried out approximately 2 hours after insertion. The average $\Delta L_{-} P$ was $+0.2 \mathrm{~cm}$ (range, $0-0.4 \mathrm{~cm}$ ) after the second treatment day, and remained on average the same during the treatment course, although the length did change in the individual catheters (Figure 2B).

The average catheter length within tissue $\left(L_{\mathrm{C}-\mathrm{B}}\right)$ was $12.3 \mathrm{~cm}$ (range, 8.1-17.4 cm). Assuming all of the catheter stretch occurred within the "in tissue" section of the catheter, the maximum stretch was on average $+1.6 \%$ (range, $0.1-2.9 \%)$. The maximum stretch of $+0.4 \mathrm{~cm}(+1.9 \%)$ was seen in five of 76 catheters. Weak correlation was seen 
A

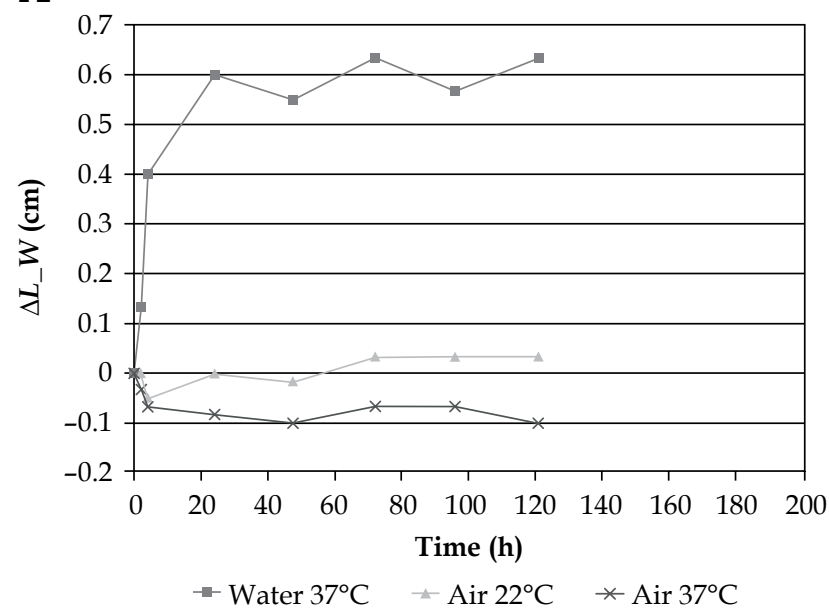

C

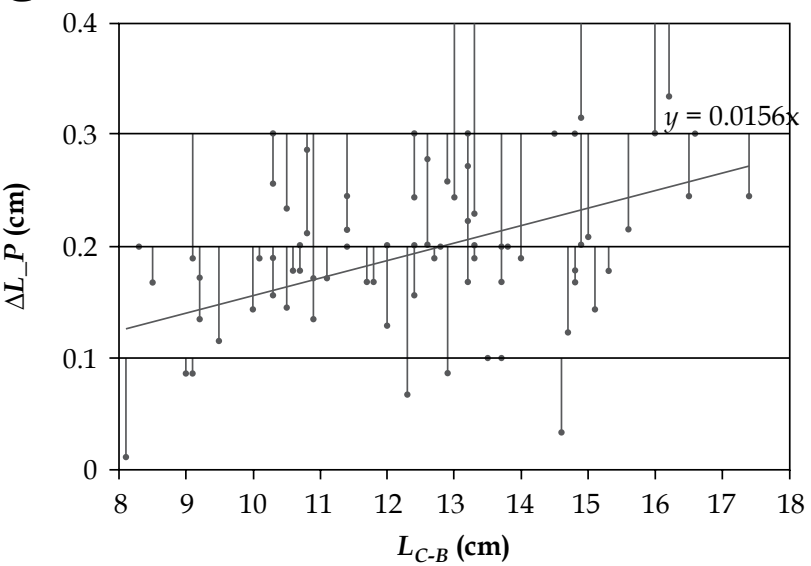

B

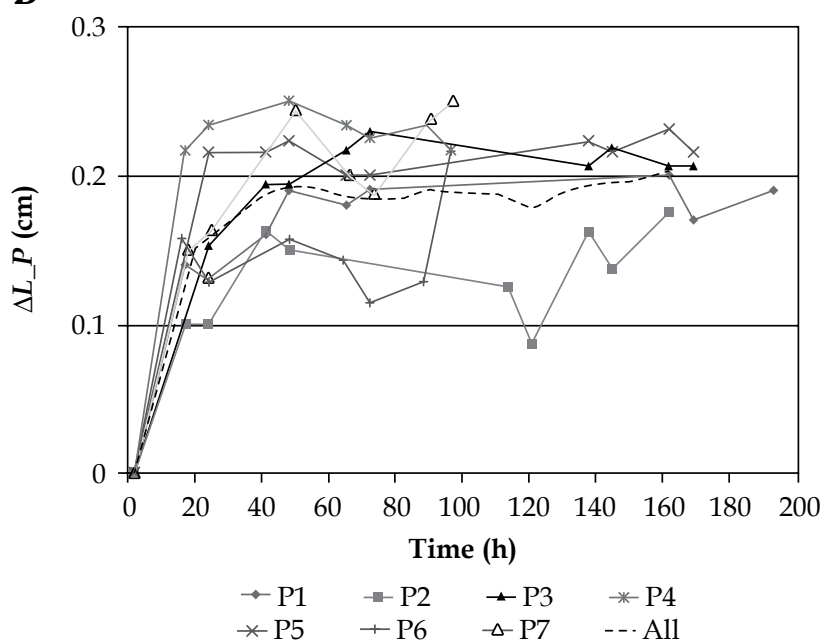

D

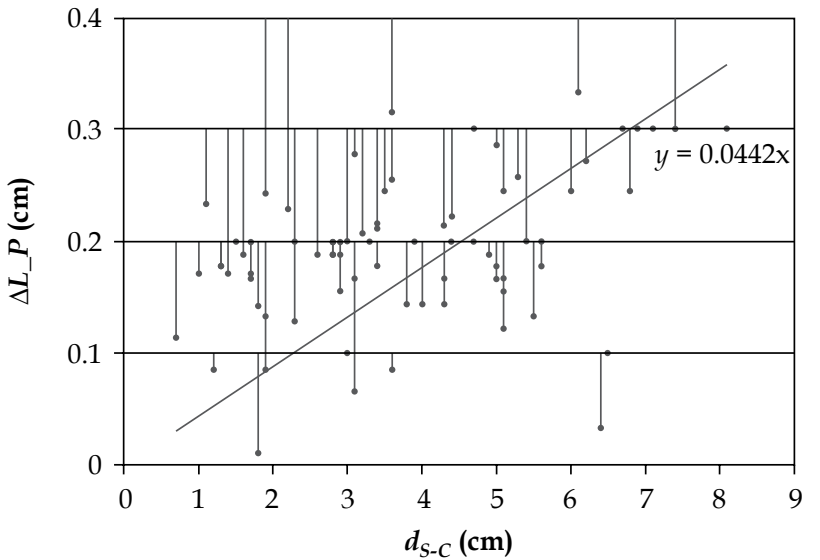

Fig. 2. A) Average length change $\Delta L_{-} W(\mathrm{~cm})$ of three catheters after the exposure to water at $37^{\circ} \mathrm{C}\left(\mathrm{Water} 37^{\circ} \mathrm{C}\right)$, to air at $22^{\circ} \mathrm{C}$ (Air $22 \mathrm{C}$ ), and to air at $37^{\circ} \mathrm{C}$ (Air $37^{\circ} \mathrm{C}$ ) with respect to the time (h). B) Average length change $\Delta L \_P$ of all catheters per patient (P1-P7) and for all patients (All) two hours after insertion with respect to the time (h). Average $\Delta L \_P(\bullet)$ for each catheter per treatment course with respect to catheter length in tissue $L_{C-B}(\mathrm{~cm})(\mathbf{C})$ or with respect to catheter depth from the skin $d_{S-C}(\mathrm{~cm})(\mathbf{D})$. The error bars present the minimum and maximum $\Delta L_{-} P$ for each catheter within treatment course. If no error bars are present, $\Delta L \_P$ was constant after the first length measurement. The lines present the linear fit (crossing vertical axis in zero) to average $\Delta L \_P$ values

between the average $\Delta L \_P$ per catheter and both $L_{\mathrm{C}-\mathrm{B}}$ $(\rho=0.36, p=0.0007)$ and $d_{\mathrm{S}-\mathrm{C}}(\rho=0.42, p<0.0001)$ (Figure $2 \mathrm{~B}-\mathrm{C})$.

\section{Catheter stretch applied to treatment plans}

The results for the dosimetric parameters are presented in Table 1 for $P_{R^{\prime}} P_{\Delta L_{-} P}$ and $P_{\Delta L_{-} W}$ plans. All data was shown to be normally distributed $(p<0.05)$. The average $\mathrm{D}_{90 \mathrm{CTV}}$ decreased by $1.5 \%(p<0.05)$ and $8.2 \%(p<0.05)$ for $P_{\Delta L_{-} P}$ and $P_{\Delta L_{-} W}$ plans, when compared to $P_{R}$ plans, respectively. For one patient, the $D_{0.2 c c s k i n}$ objective was exceeded already in initial plan being $102.8 \%$, which then increased to $104.0 \%$ and $111.5 \%$ for $P_{\Delta L_{-} P}$ and $P_{\Delta L_{-} W}$ plans, respectively. All other OARs remained within tolerance for both $P_{\Delta L_{-} P}$ and $P_{\Delta L_{-} W}$ plans.

\section{Discussion}

The catheter internal length measurements demonstrate that on average, a $4.0 \%$ or $1.6 \%$ length increase can be seen in nylon $6 / 6$ interstitial catheters, when catheters are exposed to water at $37^{\circ} \mathrm{C}$ or during an APBI HDR brachytherapy treatment course, respectively. The maximum stretch seen in vitro was reached faster (on average within 24 hours) than that for the patients (on average within 48 hours). The stretch seen in the catheters inserted into patients is relatively smaller than the stretch seen in vitro measurements. This could be explained partly by the difference in the initial measurements. In patients, the catheter internal length measurements were performed approximately two hours after insertion following our current practice to wait for the patient to recover from their anesthesia. This is in contrast to the initial in vitro 
measurements, which were carried out immediately before the catheters were submerged in water.

The in vitro measurements showed a $1.3 \%$ stretch after two hours of water exposure. The hypotheses that a similar stretch could also happen in actual patients within two hours, was tested for one APBI HDR brachytherapy patient, in which the catheter internal lengths were measured immediately after the insertion and then two hours after the insertion without the transfer guide tubes connected. On average, the stretch was $1.1 \%$ of the length of the catheters within tissue after two hours. Applying this initial stretch to our patient data results in total an average $2.7 \%$ or maximum $3.0 \%$ stretch during the APBI HDR brachytherapy treatment course, if the initial measurements had been performed immediately after applicator insertion.

One hypothesis to explain the increase in catheter length relates to the properties of nylon 6/6. Nylon 6/6 is hygroscopic and moisture absorption can lead to an increase in dimensions of up to 3\% [7]. Moisture absorption over time from surrounding breast tissue or seroma fluid may lead to dimensional increases resulting in catheter length measurements that are greater than expected. We found that the average maximum catheter stretch was $1.9 \%$ in clinical cases and $4.0 \%$ in our in vitro measurements, which supports this hypothesis when compared to the material specifications.

In addition to moisture absorption, mechanical tension might also be present on the treatment catheters during a course of HDR APBI due to breast tissue swelling and/or movement. This mechanical tension may also contribute to catheter stretch. The amount of tension present in clinical cases is difficult to estimate, and for these reasons, it was omitted from the in vitro experiment. In addition, the in vitro experiment did not include exposure to water at room temperature $\left(22^{\circ} \mathrm{C}\right)$ as this was not thought to add any significant information related to phenomena seen in clinical cases, in which the catheters are exposed only to body temperature.

In this study, a $0.5 \mathrm{~cm}$ expansion around the CTV was used to determine dwell volume. This resulted in dwell positions at least $0.25 \mathrm{~cm}$ outside of the CTV in the direction of the catheters. This was considered to improve robustness for catheter inter-fraction variation and catheter stretching. After applying the maximum $\Delta L_{-} P$ and $\Delta L \_W$ seen in each catheter to the treatment plans, our results show approximately $1.5 \%$ and $8.2 \%$ statistically significant $\mathrm{D}_{90 \mathrm{C} T \mathrm{v}}$ dose reduction, respectively. One would assume that if a tighter dwell volume had been applied, a larger dose reduction might have been seen in $\mathrm{D}_{90 \mathrm{CTV}}$. It is considered that a $1.5 \%$ decrease in dose coverage is not significant in terms of patient outcome and in relation with other uncertainties present such as catheter inter-fraction motion and dose calculation accuracy. However, an $8.2 \%$ decrease may have a clinical impact and could be possible at centers where the catheters are measured only immediately after the insertion. For these reasons, additional length checks are recommended during APBI HDR brachytherapy treatment, and applicator lengths can be adjusted in the treatment plan accordingly. Otherwise possible catheter stretch after insertion should be taken into account.
Our institution has a long history of internal length measurements for other applicators connected to the same transfer guide tubes that are used for these breast treatments and none of these have previously shown any stretching from the known length (tolerance of $\pm 0.1 \mathrm{~cm}$ ). The measurement wire slides easily into plastic interstitial catheters and does not contribute any additional force that would stretch the catheters. The internal length measurements are always done prior to each treatment as recommended by the manufacturer of our ${ }^{192}$ Ir remote afterloader (VariSource iX, Varian Medical Systems). This method is efficient in seeing any internal length change in catheters.

In our clinical practice, the usual tolerance for catheter length measurements is $\pm 0.1 \mathrm{~cm}$. For this technique, we propose adding an extra $0.1 \mathrm{~cm}$ to the applicator length for each catheter in the treatment plan. This is justified by the fact that on average each catheter stretched by $0.2 \mathrm{~cm}$, so the variation from the planned length will usually be within $0.1 \mathrm{~cm}$. If no stretch occurs post-planning then the planned length and actual length should still be within $0.1 \mathrm{~cm}$. In addition, for this technique, we will now allow a tolerance for catheter length measurement of $\pm 0.2 \mathrm{~cm}$ from the planned value. This will allow up to a $0.3 \mathrm{~cm}$ catheter stretch without any corrective action taken. If catheters stretch more than $0.3 \mathrm{~cm}$, a treatment plan adjustment will be considered.

A limitation of this study is that the maximum $\Delta L_{-} P$ per catheter was introduced to the initial plans, and it was assumed that this maximum stretch occurred for all fractions. In all cases, the maximum stretch was not present for fraction one and tended to change over time. Furthermore, it is unknown whether this catheter stretch occurs evenly over the full catheter length inside tissue, or is dependent on some other function of the implant such as seroma site. By applying the "worst case scenario" for each catheter, rather than using the average stretch, we have confidence that the reduction in $\mathrm{D}_{90 \mathrm{CTV}}$ due to catheter stretch is lower than reported here and likely to be of little clinical significance. Despite this, we have implemented the aforementioned change in our practice to reduce this error even further. Weak correlation was shown between the average $\Delta L \_P$ and both the catheter length inside tissue $L_{C-B}$ and the mid-catheter distance from skin $\mathrm{d}_{\mathrm{S}-\mathrm{C} \text {. This }}$ may indicate that even larger $\Delta L_{-} P$ values may be seen for patients with large breasts and deep target locations.

Kandasamy et al. [8] studied inter-fraction variation during a course of interstitial HDR brachytherapy for various treatment sites, including multi-catheter breast brachytherapy. For breast treatment with rigid needles, the average inter-fraction catheter displacement was $<0.3 \mathrm{~cm}$ resulting in an average $\mathrm{D}_{90 \mathrm{CTV}}$ reduction of $3 \%$. These results may not be directly transferable to our technique due to differences in the type of catheter used. Kim [9] studied the source-position uncertainty in HDR balloon brachytherapy for breast cancer and concluded that for the balloon applicator, $\mathrm{a} \pm 0.2 \mathrm{~cm}$ tolerance for source position accuracy was acceptable in most clinical cases. Again, these results cannot be directly compared to our technique due to differences in applicator and target volume definition. Another limitation of our study 
is that other factors influencing inter-fraction variation, such as positional displacement of catheters due to seroma changes, tissue deformation, or inflammation, were not included in the dosimetric analysis. Serial CT imaging over a patient's treatment course as well as details on catheter stretch are needed to determine the full dosimetric impact of inter-fraction variation with this technique.

\section{Conclusions}

Nylon 6/6 catheters were seen to stretch when exposed to water and during a course of multi-catheter interstitial breast brachytherapy. The observed length increase was shown to have minor dosimetric impact in clinical cases when a $0.5 \mathrm{~cm}$ dwell volume selection expansion was applied to the treatment plans and the initial length measurements were done two hours after the catheter insertion. In these cases, we propose reducing the already small dosimetric impact of catheter stretch by adding an extra $0.1 \mathrm{~cm}$ to the original applicator length in the treatment planning. In institutions where catheters are cut to a known length immediately after the insertion or the length measurements are only done immediately after the insertion, the catheter stretch may have dosimetrical impact and additional catheter length checks are recommended.

\section{Disclosure}

Authors report no conflict of interest.

\section{References}

1. Shaitelman SF, Amendola B, Khan A et al. American Brachytherapy Society Task Group Report: Long-term control and toxicity with brachytherapy for localized breast cancer. Brachytherapy 2017; 16: 13-21.

2. Lettmaier S, Kreppner S, Lotter M et al. Radiation exposure of the heart, lung and skin by radiation therapy for breast cancer: a dosimetric comparison between partial breast irradiation using multicatheter brachytherapy and whole breast teletherapy. Radiother Oncol 2011; 100: 189-194.

3. Major T, Gutiérrez C, Guix B et al. Recommendations from GEC ESTRO Breast Cancer Working Group (II): Target definition and target delineation for accelerated or boost partial breast irradiation using multicatheter interstitial brachytherapy after breast conserving open cavity surgery. Radiother Oncol 2016; 18: 199-204.

4. Strnad V, Ott OJ, Hildebrandt G et al. 5-year results of accelerated partial breast irradiation using sole interstitial multicatheter brachytherapy versus whole-breast irradiation with boost after breast-conserving surgery for low-risk invasive and in-situ carcinoma of the female breast: a randomised, phase 3, non-inferiority trial. Lancet 2016; 387: 229-238.

5. Valentin J; International Commission on Radiation Protection. Prevention of high-dose-rate brachytherapy accidents. ICRP Publication 97. Ann ICRP 2005; 35: 1-51.

6. Strnad V, Hannoun-Levi JM, Guinot JL et al. Recommendations from GEC ESTRO Breast Cancer Working Group (I): Target definition and target delineation for accelerated or boost Partial Breast Irradiation using multicatheter interstitial brachytherapy after breast conserving closed cavity surgery. Radiother Oncol 2015; 115: 342-348.

7. GoodFellow: Material information for Polyamide - Nylon 6,6 (PA 6,6). Available at: http://www.goodfellow.com/E/Poly amide-Nylon-6-6.html [Accessed: 15 March 2017].
8. Kandasamy S, Reddy KS, Nagarajan V et al. Inter-fraction variation in interstitial high-dose-rate brachytherapy. J Radiother Pract 2015; 14: 143-151.

9. Kim Y. Dosimetric impact of source-positioning uncertaintyin high-dose-rate balloon brachytherapy of breast cancer. J Contemp Brachytherapy 2015; 7: 387-396. 